\title{
A Novel Herbal Medicine for Dyslipidemia: Assessments in Experimental Models
}

\author{
Pham Ba Tuyen, ${ }^{1}$ Truong Thi Huyen, ${ }^{1}$ Dinh Thi Thu Hang, ${ }^{2}$ and Pham Thi Van Anh $\mathbb{D}^{2}$ \\ ${ }^{1}$ Traditional Medicine Hospital, Ministry of Public Security, Hanoi, Vietnam \\ ${ }^{2}$ Hanoi Medical University, Hanoi, Vietnam \\ Correspondence should be addressed to Pham Thi Van Anh; phamvananh@hmu.edu.vn
}

Received 9 January 2021; Revised 29 March 2021; Accepted 10 April 2021; Published 22 April 2021

Academic Editor: Valeria Sülsen

Copyright (C) 2021 Pham Ba Tuyen et al. This is an open access article distributed under the Creative Commons Attribution License, which permits unrestricted use, distribution, and reproduction in any medium, provided the original work is properly cited.

\begin{abstract}
Dyslipidemia substantially contributes to the risk of cardiovascular diseases. The polyherbal formulation has been a traditional therapeutic strategy used to treat dyslipidemia. This study was designed to evaluate the effects of a novel herbal medicine called "GANMO" on an experimental animal model with endogenous dyslipidemia and exogenous dyslipidemia. In the endogenous hyperlipidemia model, rats were previously treated with GANMO tablets and intraperitoneally injected with poloxamer 407 to induce hyperlipidemia. In the exogenous hyperlipidemia model, rats were given oral administration of oil-cholesterol mixture and GANMO for 4 consecutive weeks. Serum lipid profiles were assessed at all experimental animals. In both models, GANMO at both doses significantly decreased the serum total cholesterol (TC) level and non-high-density lipoprotein (HDL) cholesterol level as compared with the model group. HDL cholesterol levels increased in rats with high doses of GANMO compared to those with low doses. GANMO at both doses substantially reduced TG level in the endogenous hyperlipidemia model. In conclusion, GANMO tablets posed a positive effect on serum lipid modulations in dyslipidemia models.
\end{abstract}

\section{Introduction}

Dyslipidemia refers to the excess status of fatty substances including cholesterol, triglyceride, and decreased highdensity lipoprotein cholesterol (HDL-C) in the bloodstream [1]. Besides, dyslipidemia is a major contribution to the onset of cardiovascular diseases (CVD) such as atherosclerosis, myocardial infarction, and cerebral vascular accidents, which are the main causes of the global burden of diseases [2]. Currently, although statins (e.g., simvastatin, atorvastatin, and rosuvastatin) have been widely used to reduce plasma lipids, their usage may be limited due to their side effects such as hepatotoxicity, rhabdomyolysis, or skeletal muscle injury [3]. Thus, natural alternatives have been proposed to control lipid metabolism.

Using multiple herbs (i.e., polyherbal) has been used in the traditional medicine approach given that it can improve the effects of therapy in curing a specific disease [4]. Several previous studies in Asian countries such as Indonesia [5] or
China [6] have attempted to produce polyherbal medicine to treat dyslipidemia. In Vietnam, we have produced a novel polyherbal medicine called "GANMO" in improving serum lipid profiles. Each drug tablet contains Pericarpium Citri Reticulatae, Atractylodes macrocephala Koidz., Reynoutria japonica Houtt., Folium Nelumbinis, Sargassum pallidum (Tum), Cassia tora L., Alisma plantago aquatica L., Fallopia multiflora (Thunb.) Haraldson, Crataegus cuneara Sied. et Zucc., and Rheum palmatum L. Several previous studies have shown the benefits of these herbal medicines in reducing serum lipid levels [7-9]. This study aimed to assess the effects of GANMO tablets on serum lipid profiles of dyslipidemia experimental animals.

\section{Materials and Methods}

2.1. Preparation of Materials. GANMO was prepared and offered in form of tablets, which contained Pericarpium Citri Reticulatae $0.83 \mathrm{~g}$, Atractylodes macrocephala Koidz. $1 \mathrm{~g}$, 
Reynoutria japonica Houtt. $1.25 \mathrm{~g}$, Folium Nelumbinis $1.25 \mathrm{~g}$, Sargassum pallidum (Tum) $1.25 \mathrm{~g}$, Cassia tora L. $1.25 \mathrm{~g}$, Alisma plantago aquatica L. $1 \mathrm{~g}$, Fallopia multiflora (Thunb.) Haraldson $1 \mathrm{~g}$, Crataegus cuneara Sied. et Zucc. $0.83 \mathrm{~g}$, and Rheum palmatum L. $0.83 \mathrm{~g}$. The tables were produced in the Traditional Medicine Hospital, Ministry of Public Security, Hanoi, Vietnam. The recommended dosage was 12 tablets per day.

The materials were prepared in compliance with the standards of Vietnamese Pharmacopoeia V. Grinding and filtering material procedures were implemented under the microbiological safety and Pharmacopoeia V requirements. Peels of Pericarpium Citri Reticulatae, roots of Atractylodes macrocephala Koidz., fruits of Reynoutria japonica Houtt., leaves of Folium Nelumbinis, Sargassum pallidum (Tum), seeds of Cassia tora L., roots of Alisma plantago aquatica L., roots of Fallopia multiflora (Thunb.) Haraldson, fruits of Crataegus cuneara Sied. et Zucc., and roots of Rheum palmatum $L$. were washed, removed impurities, and cooked twice. At the first time, materials were cooked for 8 hours from boiling, collecting the extract (I), and adding water. At the second time, materials were cooked for 2 hours from boiling and collecting the extract (II). Extracts (I) and (II) were put together and concentrated at temperature $60^{\circ} \mathrm{C}$.

2.2. Animal Preparation. Wistar rats (males and females, weighing $180-220 \mathrm{~g}$ ) were provided by the Center of Experimental Animals, DanPhuong, Hanoi, Vietnam. Healthy Swiss albino mice (of both sexes, weighing 21-25g) were originated from the National Institute of Hygiene and Epidemiology (NIHE), Vietnam. Animals were housed under the standard environment (temperature: $25^{\circ} \mathrm{C} \pm 2^{\circ} \mathrm{C}$ and relative humidity: $80 \% \pm 10 \%$ ), 12 -hour dark/light time (lights on at 6 : $00 \mathrm{AM})$. We fed the mice with standard animal feed and allowed free access to water. After randomizing the rats into different intervention groups as well as before implementing the experiment, mice were transferred to the laboratory conditions for 7 days at the laboratory of Pharmacology Department, Hanoi Medical University. All protocols used in this study were approved by the Scientific Board Committee of Hanoi Medical University (ref. number: IRB00003121).

2.3. Chemical Preparation. In this study, we used propylthiouracil (Rieserstat ${ }^{\circledR}$ ) $50 \mathrm{mg}$, cholesterol (Merk, Germany), cholic acid, poloxamer 407, (Sigma, Singapore), atorvastatin $10 \mathrm{mg}$ (Stellapharm J.V. Co., Ltd.), and peanut oil (Vietnam). Biochemical analyzer (ERBA Chem, India) and commercial ERBA diagnostic kits were used for serum analysis of total cholesterol (TC), triglyceride (TG), and high-density lipoprotein-cholesterol (HDL-C).

2.4. Procedure. Exogenous dyslipidemia model: dyslipidemia was induced in rats by oral administration of cholesterol mixture (cholesterol $10 \%$, cholic acid $1 \%$, propylthiouracil (PTU) $0.5 \%$, and peanut oil added to precisely $1 \mathrm{~mL}$ ) for four weeks [10]. Wistar Albino rats (180-220 g) were divided into 5 groups with 10 rats per group. Rats were given oral medication twice per day, at least two hours apart:
Group 1 (control group): distilled water $10 \mathrm{~mL} / \mathrm{kg}$ b.w twice per day

Group 2 (model group): cholesterol mixture $10 \mathrm{~mL} / \mathrm{kg}$ b.w per day and distilled water $1 \mathrm{~mL} / 100 \mathrm{~g}$ b.w per day Group 3 (atorvastatin): cholesterol mixture $10 \mathrm{~mL} / \mathrm{kg}$ b.w per day and atorvastatin with the dose of $10 \mathrm{mg} / \mathrm{kg}$ b.w per day

Group 4 (GANMO-low dose): cholesterol mixture $10 \mathrm{~mL} / \mathrm{kg}$ b.w per day and GANMO at the dose of $15.1 \mathrm{~g} / \mathrm{kg}$ b.w per day (equivalent to clinical dose)

Group 5 (GANMO-high dose): cholesterol mixture $10 \mathrm{~mL} / \mathrm{kg}$ b.w per day and GANMO at the dose of $45.3 \mathrm{~g} / \mathrm{kg}$ b.w per day (three times as high as clinical dose)

Rat's body weight was recorded at baseline, after 2 weeks, and after 4 weeks. On day 15 and day 29, rats fasted overnight. Blood was collected to measure serum TC, TG, and HDLconcentrations. LDL-C concentration was calculated using the Friedewald formula: LDL-C $=$ TC $-($ HDL-C) $-(T G / 2.2)$ $(\mathrm{mmol} / \mathrm{L})$ [11].

Endogenous dyslipidemia model: poloxamer 407(P-407-) induced dyslipidemia model was described by Millar et al. [12]. Our mice were randomly separated into five groups with 10 rats per group.

Group 1 (control group): mice were given oral distilled water $1 \mathrm{~mL} / 100 \mathrm{~g}$ b.w/day and then received intraperitoneal (IP) injection $0.9 \% \mathrm{NaCl} 10 \mathrm{~mL} / \mathrm{kg}$ b.w on day 7 .

Group 2 (model group): mice were given oral distilled water $1 \mathrm{~mL} / 100 \mathrm{~g}$ b.w/day and then received IP injection $2 \%$ P-407 at the dose of $200 \mathrm{mg} / \mathrm{kg}$ b.w on day 7

Group 3 (atorvastatin): mice were given oral atorvastatin at the dose of $100 \mathrm{mg} / \mathrm{kg}$ b.w/day and then received IP injection 2\% P-407 at the dose of $200 \mathrm{mg} / \mathrm{kg}$ b.w on day 7 Group 4 (GANMO-low dose): mice were given oral GANMO at the dose of $30.2 \mathrm{~g} / \mathrm{kg}$ b.w/day (equivalent to clinical dose); then received IP injection 2\% P-407 at the dose of $200 \mathrm{mg} / \mathrm{kg}$ b.w on day 7 .

Group 5 (GANMO-high dose): mice were given per oral GANMO at the dose of $90.6 \mathrm{~g} / \mathrm{kg}$ b.w/day (3 times as high as clinical dose) and then injected i.p. 2\% P-407at the dose of $200 \mathrm{mg} / \mathrm{kg}$ b.w on day 7 .

Blood was collected at $24 \mathrm{~h}$ after IP injection of P-407 and analyzed for serum lipids including TG, TC, and HDL-C. Non-HDL-cholesterol (non-HDL-C) was estimated: non-HDL-C $=$ TC $-(\mathrm{HDL}-\mathrm{C})$.

2.5. Statistical Analysis. Data were analyzed using SPSS software version 20.0. T-test and ANOVA tests were performed to examine the differences in serum lipid profiles in different groups. A $p$ value $<0.05$ was considered to be statistically significant.

\section{Results}

3.1. Effects of GANMO Tablets on Lipid Levels in Exogenous Dyslipidemia Model. Table 1 shows that, for all time points, 
TABLE 1: Effect of GANMO tablets on body weight gain in dyslipidemia.

\begin{tabular}{lcccc}
\hline Groups & $n$ & Initial weight $(\bar{X} \pm$ SD $)$ & After 2 weeks $(\bar{X} \pm$ SD $)$ & After 4 weeks $(\bar{X} \pm$ SD $)$ \\
\hline Control & 10 & $205.00 \pm 35.10$ & $206.00 \pm 25.03$ & $224.00 \pm 42.48$ \\
Model & 10 & $214.00 \pm 32.04$ & $225.00 \pm 22.61$ & $206.00 \pm 21.19$ \\
Atorvastatin $(10 \mathrm{mg} / \mathrm{kg})$ & 10 & $213.00 \pm 56.18$ & $208.50 \pm 56.18$ & $208.00 \pm 47.56$ \\
GANMO $(15.1 \mathrm{~g} / \mathrm{kg})$ & 10 & $204.00 \pm 37.48$ & $218.00 \pm 41.04$ & $204.50 \pm 48.10$ \\
GANMO $(45.3 \mathrm{~g} / \mathrm{kg})$ & 10 & $222.00 \pm 43.92$ & $231.50 \pm 53.85$ & $220.00 \pm 66.16$ \\
\hline
\end{tabular}

no significant difference in body weight was found among groups $(p>0.05)$.

Table 2 shows that atorvastatin tended to decreased levels of TG, TC, and LDL-C, and GANMO at the dose of $45.3 \mathrm{~g} / \mathrm{kg}$ had a tendency to reduce TG level as compared with the model group after 2 weeks of treatment, but no significant change was observed $(p>0.05)$.

Figure 1 and Table 3 show that, after 4 weeks of treatment, in the groups treated with atorvastatin and GANMO, there was a considerable decrease in levels of LDL-C and TC as compared with the model group. In terms of the HDL-C level, the group treated with GANMO at the dose of $45.3 \mathrm{~g} /$ $\mathrm{kg}$ tended to increase the HDL-C level, but no significant difference was observed $(p>0.05)$. No differential change was observed between groups treated with GANMO at the dose of $15.1 \mathrm{~g} / \mathrm{kg}$ and the model group $(p>0.05)$.

3.2. Effects of GANMO on Lipid Levels in Poloxamer 407Induced Dyslipidemia. Table 4 illustrated that there was a dramatic development in all lipid levels in the model group as compared with the control group $(p<0.001)$.

Table 5 shows that there was a substantial reduction in the TC level and non-HDL-C level and a significant increase in HDL-C level in the group treated atorvastatin as compared with the model group. GANMO at both doses of $90.6 \mathrm{~g} / \mathrm{kg}$ b.w and $30.2 \mathrm{~g} / \mathrm{kg}$ b.w significantly decreases TG level, TC level, and non-HDL-C level developing the HDL-C level as compared with the model group.

\section{Discussion}

In literature, the model of exogenous dyslipidemia induced by oil-cholesterol mixture (cholesterol with bile acid and hypothyroidism-inducing agents) is widely used to screen natural or synthetic drugs [13]. In this study, we evaluated the effects of GANMO tablets on the changes in serum lipid levels.

The findings of this study suggested that there was no significant difference in body weight among intervention and control groups at all time points after four weeks of the high-fat diet. However, the model of hyperlipidemia was induced successfully through the significant change of serum biochemical indexes. In particular, rats in the model group given the cholesterol-rich diet exhibited high plasma lipid levels, including TC, TG, and LDL-C levels. There was a significant improvement in serum lipid indexes in the group treated with GANMO tablets as compared with the control group after 4 weeks. The lipid-lowering effect of GANMO was a dose-dependent effect, and GANMO at a high dose posed a greater effect than the low dose.
A model of endogenous hyperlipidemia was developed by IP injection of P-407 $200 \mathrm{mg} / \mathrm{kg}$ b.w. P-407, a polyetherbased nonionic surface-active-agent (surfactant), providing an attractive means of inducing hyperlipidemia because of its rapid onset and seeming lack of over toxicity as compared with Triton WR-1339. P-407 has been known to cause significant dose-dependent hypercholesterolemia and hypertriglyceridemia in rats via several mechanisms (e.g., inhibition of lipoprotein lipase, indirect stimulation of HMG-CoA (3-hydroxy-3-methylglutarylCo-A) reductase, and promotion of concentration of hepatic cholesterol content) [14]. Based on its mechanism and shreds of evidence of efficiency, the statin is chosen as the drug reference standard. It inhibits HMG-CoA reductase, which counters the effect of P-407, thus decreases the serum TC. In addition, it also lowers the level of LDL by lowering the level of its precursor (VLDL and IDL), which further enhances its lipidlowering effect. Currently, seven different statins are available generically, but only atorvastatin and rosuvastatin are used in high-intensity therapy [3].

Because of its safety, various P-407-induced hyperlipidemia models in rats and mice have been often conducted with the standard dose of $500 \mathrm{mg} / \mathrm{kg}$. In this study, the dose of $200 \mathrm{mg} / \mathrm{kg}$ was chosen to evaluate and compare the effects of the regimens. Within $24 \mathrm{~h}$ of its IP injection, a profound hyperlipidemia state was achieved. Our finding showed that TG concentration increased substantially by 6.8 -fold, and TC levels and non-HDL-C concentrations increased by 2.9 fold and 4.1-fold, respectively. Based on the success of the P-407-induced hyperlipidemia model, the effects of the GANMO tablet could be evaluated precisely. The data indicated that there was a substantial decrease in the levels of TG, TC, and non-HDL-C and a significant increase in the HDL-C level in dyslipidemia mice treated with GANMO at both doses of $30.2 \mathrm{~g} / \mathrm{kg}$ b.w and $90.6 \mathrm{~g} / \mathrm{kg}$ b.w. Mechanisms of compounds in GANMO tablets in the treatment are very complex.

These effects of GANMO tablets suggested the combination of lipid-lowering effects of herbal medicines. Yu et al. [7] suggested that polymethoxyflavonoids (PMFs) which were isolated from Pericarpium Citri Reticulatae (PCR) could be used to reduce serum lipid levels [7]. Meanwhile, in Reynoutria japonica Houtt, there are more than 70 compounds (such as quinones, stilbenes, or flavonoids) isolated and identified. These compounds were illustrated to regulate lipid metabolism and the effect on hyperlipidemia [8]. Flavonoids (epicatechin, myricetin, hyperoxide, quercitrin, and quercetin) were extracted from Rheum palmatum $L$. [14]. These flavonoids posed lipid-lowering effects through various research studies [15-18]. 
TABLE 2: Effect of GANMO on lipid levels in cholesterol-induced dyslipidemia after 2 weeks of treatment.

\begin{tabular}{lccccc}
\hline Groups/treatment $2^{\text {nd }}$ week & $n$ & TG $(\bar{X} \pm$ SD $)$ & TC $(\bar{X} \pm$ SD $)$ & HDL-C $(\bar{X} \pm$ SD $)$ & LDL-C $(\bar{X} \pm$ SD $)$ \\
\hline Control group & 10 & $0.50 \pm 0.11$ & $2.16 \pm 0.15$ & $1.08 \pm 0.15$ & $0.85 \pm 0.22$ \\
Model group & 10 & $0.54 \pm 0.17$ & $2.38 \pm 0.35$ & $1.16 \pm 0.20$ & $1.11 \pm 0.19^{*}$ \\
Atorvastatin $(10 \mathrm{mg} / \mathrm{kg})$ & 10 & $0.49 \pm 0.12$ & $2.36 \pm 0.29$ & $1.06 \pm 0.16$ & $1.08 \pm 0.34$ \\
GANMO $(15.1 \mathrm{~g} / \mathrm{kg})$ & 10 & $0.74 \pm 0.25$ & $2.66 \pm 0.28$ & $1.05 \pm 0.22$ & $1.27 \pm 0.46$ \\
GANMO $(45.3 \mathrm{~g} / \mathrm{kg})$ & 10 & $0.45 \pm 0.14$ & $2.56 \pm 0.33$ & $0.99 \pm 0.17$ & $1.37 \pm 0.40$ \\
\hline
\end{tabular}

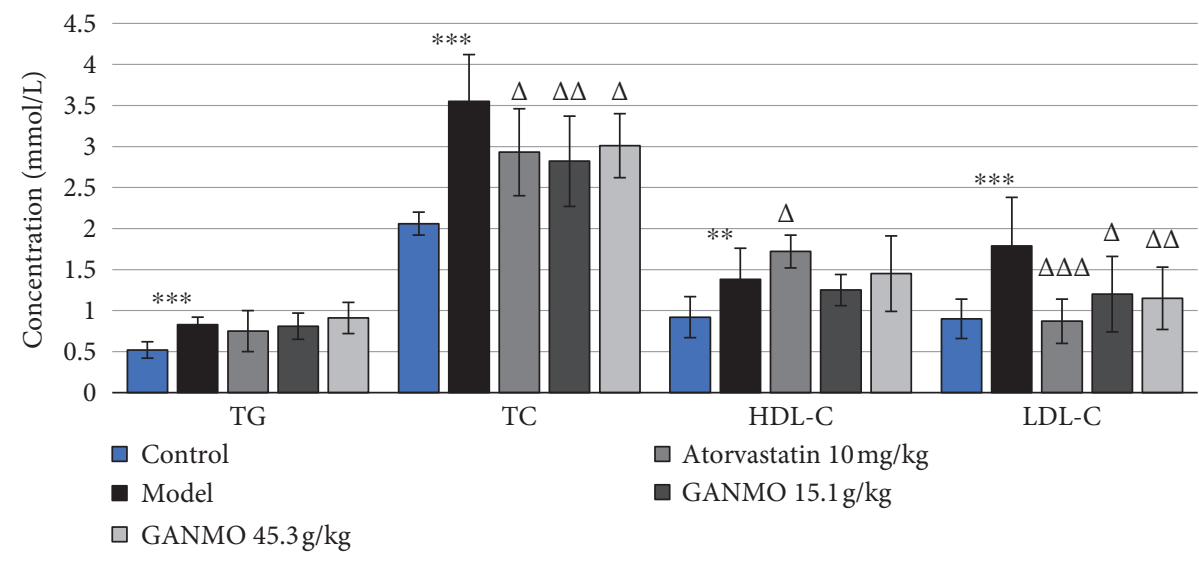

Figure 1: Changes in the serum lipid concentration of animals in cholesterol-induced dyslipidemia after 4 weeks.

TABLE 3: Effect of GANMO on lipid levels in cholesterol-induced dyslipidemia after 4 weeks of treatment.

\begin{tabular}{|c|c|c|c|c|c|}
\hline Groups/treatment on $4^{\text {th }}$ week & $n$ & $\mathrm{TG}(\bar{X} \pm \mathrm{SD})$ & $\mathrm{TC}(\bar{X} \pm \mathrm{SD})$ & HDL-C $(\bar{X} \pm S D)$ & LDL-C $(\bar{X} \pm \mathrm{SD})$ \\
\hline Control group & 10 & $0.52 \pm 0.10$ & $2.06 \pm 0.14$ & $0.92 \pm 0.25$ & $0.90 \pm 0.24$ \\
\hline Model group & 10 & $0.83 \pm 0.09^{* * *}$ & $3.55 \pm 0.57^{* * *}$ & $1.38 \pm 0.38^{* * *}$ & $1.79 \pm 0.59^{* * *}$ \\
\hline Atorvastatin $(10 \mathrm{mg} / \mathrm{kg})$ & 10 & $0.75 \pm 0.25$ & $2.93 \pm 0.53^{\Delta}$ & $1.72 \pm 0.20^{\Delta}$ & $0.87 \pm 0.27^{\Delta \Delta \Delta}$ \\
\hline GANMO $(15.1 \mathrm{~g} / \mathrm{kg})$ & 10 & $0.81 \pm 0.16$ & $2.82 \pm 0.55^{\Delta \Delta}$ & $1.25 \pm 0.19$ & $1.20 \pm 0.46^{\Delta}$ \\
\hline GANMO $(45.3 \mathrm{~g} / \mathrm{kg})$ & 10 & $0.91 \pm 0.19$ & $3.01 \pm 0.39^{\Delta}$ & $1.45 \pm 0.46$ & $1.15 \pm 0.38^{\Delta \Delta}$ \\
\hline
\end{tabular}

Note: statistical analysis was done with $t$ test and ANOVA test, and $p<0.05$ was considered to be statistically significant; ${ }^{* * * * * *}: p<0.01$ and $p<0.001$ compared with control group; ${ }^{\Delta, \Delta \Delta, \Delta \Delta \Delta}$ : $p<0.05, p<0.01$, and $p<0.001$ compared with the model group.

TABLe 4: Hyperlipidemia model induced by P-407.

\begin{tabular}{lccc}
\hline Lipid levels $(\mathrm{mmol} / \mathrm{l})$ & $n$ & Control group $(\bar{X} \pm \mathrm{SD})$ & Model group $(\bar{X} \pm \mathrm{SD})$ \\
\hline TG & 10 & $0.93 \pm 0.15$ & $6.29 \pm 1.24^{* * *}$ \\
TC & 10 & $2.36 \pm 0.51$ & $6.91 \pm 0.92^{* * *}$ \\
HDL-C & 10 & $1.05 \pm 0.23$ & $1.53 \pm 0.13^{* * *}$ \\
Non-HDL-C & 10 & $1.31 \pm 0.40$ & $5.38 \pm 0.88^{* * *}$ \\
\hline
\end{tabular}

Note: ${ }^{* * *} p<0.001$ compared with the control group.

TABle 5: Effects of GANMO on lipid levels in Poloxamer 407-induced dyslipidemia after 1 week of treatment.

\begin{tabular}{lccccc}
\hline \multirow{2}{*}{ Groups } & $n$ & \multicolumn{4}{c}{ Serum lipid levels $(\bar{X} \pm \mathrm{SD})$} \\
& & TG $(\mathrm{mmol} / \mathrm{L})$ & TC $(\mathrm{mmol} / \mathrm{L})$ & HDL-C $(\mathrm{mmol} / \mathrm{L})$ & Non-HDL-C $(\mathrm{mmol} / \mathrm{L})$ \\
\hline Model group & 10 & $6.29 \pm 1.24$ & $6.91 \pm 0.92$ & $1.53 \pm 0.13$ & $5.38 \pm 0.88$ \\
Atorvastatin $(100 \mathrm{mg} / \mathrm{kg})$ & 10 & $6.50 \pm 1.92$ & $4.70 \pm 1.04^{\Delta \Delta \Delta}(\downarrow 32.0 \%)$ & $1.63 \pm 0.19(\uparrow 6.5 \%)$ & $3.07 \pm 1.08^{\Delta \Delta \Delta}(\downarrow 42.9 \%)$ \\
GANMO $(30.2 \mathrm{~g} / \mathrm{kg})$ & 10 & $4.61 \pm 1.52^{\Delta}(\downarrow 26.7 \%)$ & $5.15 \pm 0.68^{\Delta \Delta \Delta}(\downarrow 25.5 \%)$ & $1.74 \pm 0.15^{\Delta \Delta}(\uparrow 13.7 \%)$ & $3.41 \pm 0.77^{\Delta \Delta \Delta}(\downarrow 36.6 \%)$ \\
GANMO $(90.6 \mathrm{~g} / \mathrm{kg})$ & 10 & $3.78 \pm 0.72^{\Delta \Delta \Delta}(\downarrow 39.9 \%)$ & $5.04 \pm 1.04^{\Delta \Delta \Delta}(\downarrow 27.1 \%)$ & $1.71 \pm 0.21^{\Delta}(\uparrow 11.8 \%)$ & $3.33 \pm 0.93^{\Delta \Delta \Delta}(\downarrow 38.1 \%)$ \\
\hline
\end{tabular}

Note: statistical analysis was done with $t$-test and ANOVA test, and $p<0.05$ was considered to be statistically significant; ${ }^{* * * * * *}: p<0.01$ and $p<0.001$ compared with the control group; ${ }^{\Delta, \Delta \Delta, \Delta \Delta \Delta}: p<0.05, p<0.01$, and $p<0.001$ compared with the model group. 


\section{Conclusion}

In conclusion, our results demonstrated that oral administration of GANMO at doses of $30.2 \mathrm{~g} / \mathrm{kg}$ and $90.6 \mathrm{~g} / \mathrm{kg}$ for consecutive 7 days significantly reduced levels of TC, TG, non-HDL-C and increased the HDL-C level in P-407-induced hyperlipidemic mice. The exogenous dyslipidemia model induced by oil-cholesterol mixture and oral administration of GANMO tablets for 4 weeks at both doses of $15.1 \mathrm{~g} / \mathrm{kg}$ and $45.3 \mathrm{~g} / \mathrm{kg}$ reduced TC level and non-HDL-C level in rats. Moreover, GANMO tablets at the dose of $45.3 \mathrm{~g} /$ $\mathrm{kg}$ tended to increase HDL-C level as compared with the model group.

\section{Data Availability}

The data used to support the findings of this study are available from the corresponding author upon request.

\section{Conflicts of Interest}

The authors declare that there are no conflicts of interest regarding the publication of this paper.

\section{References}

[1] D. J. Rader and H. H. Hobbs, "Disorders of lipoprotein metabolism," Harrisons Principles of Internal Medicine, vol. 16, p. 2286, 2005.

[2] N. A. Ciffone and T. Copple, "Managing dyslipidemia for CVD prevention," The Nurse Practitioner, vol. 44, no. 1, pp. 8-16, 2019.

[3] B. A. Golomb and M. A. Evans, "Statin adverse effects," American Journal of Cardiovascular Drugs, vol. 8, no. 6, pp. 373-418, 2008.

[4] C.-T. Che, Z. Wang, M. Chow, and C. Lam, "Herb-herb combination for therapeutic enhancement and advancement: theory, practice and future perspectives," Molecules, vol. 18, no. 5, pp. 5125-5141, 2013.

[5] W. R. Pratiwi, E. N. Sholikhah, D. A. A. Nugrahaningsih, M. M. Yuniyanti, and M. Mustofa, "Effects of poly-herbal tablet as herbal medicine on lipid level," Majalah Obat Tradisional, vol. 24, no. 1, pp. 47-51, 2019.

[6] M. Guo, Y. Liu, Z.-Y. Gao, and D.-Z Shi, "Chinese herbal medicine on dyslipidemia: progress and perspective," Evidence-Based Complementary and Alternative Medicine, vol. 2014, Article ID 163036, 11 pages, 2014.

[7] J. J. Yu, J. Su, M. Q. Yan, Z. H. Lou, and G. Y. Lyu, "[Correlation between lipid-lowering efficacy and components of pericarpium citri reticulatae]," Zhongguo Zhong Yao Za Zhi= Zhongguo Zhongyao Zazhi= China Journal of Chinese Materia Medica, vol. 44, no. 15, pp. 3335-3342, 2019.

[8] J. Patocka, Z. Navratilova, and M. Ovando-Martínez, "Biologically active compounds of knotweed (Reynoutria spp.)," Military Medical Science Letters, vol. 86, pp. 17-31, 2017.

[9] W. Xie, D. Xing, Y. Zhao et al., "A new tactic to treat postprandial hyperlipidemia in diabetic rats with gastroparesis by improving gastrointestinal transit," European Journal of Pharmacology, vol. 510, no. 1-2, pp. 113-120, 2005.

[10] M. Nassiri-Asl, F. Zamansoltani, E. Abbasi, M. M. Daneshi, and A. A. Zangivand, "Effects of Urtica dioica extract on lipid profile in hypercholesterolemic rats," Journal of Chinese Integrative Medicine, vol. 7, no. 5, pp. 428-433, 2009.

[11] W. T. Friedewald, R. I. Levy, and D. S. Fredrickson, "Estimation of the concentration of low-density lipoprotein cholesterol in plasma, without use of the preparative ultracentrifuge," Clinical Chemistry, vol. 18, no. 6, pp. 499-502, 1972.

[12] J. S. Millar, D. A. Cromley, M. G. McCoy, D. J. Rader, and J. T. Billheimer, "Determining hepatic triglyceride production in mice: comparison of poloxamer 407 with Triton WR-1339," Journal of Lipid Research, vol. 46, no. 9, pp. 2023-2028, 2005.

[13] H. Mir, D. Krouf, N. Taleb-Dida, S. Berzou, A. Guenzet, and H. Khelladi, "Effects of citrus latifolia extract on dyslipidemia and tissues redox status in rats fed a high-cholesterol diet," Nutrition \& Food Science, vol. 49, no. 6, pp. 989-999, 2019.

[14] T. P. Johnston, T. A. Korolenko, and A. Sahebkar, "P-407induced mouse model of dose-controlled hyperlipidemia and atherosclerosis: 25 years later," Journal of Cardiovascular Pharmacology, vol. 70, no. 5, pp. 339-352, 2017.

[15] A. D. Mariee, G. M. Abd-Allah, and H. A. El-Beshbishy, "Protective effect of dietary flavonoid quercetin against lipemic-oxidative hepatic injury in hypercholesterolemic rats," Pharmaceutical Biology, vol. 50, no. 8, pp. 1019-1025, 2012.

[16] S. Juźwiak, J. Wójcicki, K. Mokrzycki et al., "Effect of quercetin on experimental hyperlipidemia and atherosclerosis in rabbits," Pharmacological Reports: PR, vol. 57, no. 5, pp. 604-609, 2005.

[17] H. Cheng, N. Xu, W. Zhao et al., “(-)-Epicatechin regulates blood lipids and attenuates hepatic steatosis in rats fed highfat diet," Molecular Nutrition \& Food Research, vol. 61, no. 11, 2017.

[18] Z. Zhang, D. Zhang, B. Du, and Z. Chen, "Hyperoside inhibits the effects induced by oxidized low-density lipoprotein in vascular smooth muscle cells via oxLDL-LOX-1-ERK pathway," Molecular and Cellular Biochemistry, vol. 433, no. 1-2, pp. 169-176, 2017. 